\title{
Dark Energy and Dark Matter in Stars Physic
}

\author{
Plamen Fiziev* \\ Jount Institute of Nuclear Research, Dubna, Russian Federation \\ E-mail: Eizievdtheor.jinr.ru
}

We present the basic equations and relations for the relativistic static spherically symmetric stars (SSSS) in the model of minimal dilatonic gravity (MDG) which is locally equivalent to the $\mathrm{f}(\mathrm{R})$ theories of gravity and gives an alternative description of the effects of dark matter and dark energy. The results for the simplest form of the relativistic equation of state (EOS) of neutron matter are represented. Our approach overcomes the well-known difficulties of the physics of SSSS in the $f(R)$ theories of gravity introducing two novel EOS for cosmological energy-pressure densities and dilaton energy-pressure densities, as well as proper boundary conditions.

Frontiers of Fundamental Physics 14

15-18 July 2014

Aix Marseille University (AMU) Saint-Charles Campus, Marseille, France

${ }^{*}$ Speaker. 


\section{Introduction}

One of the most important lessons from the spectacular development of cosmology in the last fifteen years is the clear understanding that the Einstein general relativity (GR) and standard particle model (SPM) are insufficient to explain all observed phenomena in the Nature. There exist three possible ways for further development:

1) To add some new content of the Universe beyond the SPM, like dark matter and dark energy;

2) To change the theory of gravity. The simplest models are $f(R)$ [四] and MDG [0, []];

3) Some mixture of these two possibilities is not excluded by the current observational data.

Dozens of models with different functions $f(R)$ exist, some of them dubbed valuable [四].

The situation in star physics is similar. More than sixty-year development so far has not solved the problem with realistic EOS of compact star matter. At present one can find several dozens of EOS in the literature.

A series of attempts to use $f(R)$ models of gravity adopted to star physics also exist [四]. No fully convincing final result was reached.

The main goal of the present paper is to create a clear physical basis for application of MDG in star physics and thus to facilitate construction of models, which permit unified treatment of the physical problems at very different scales: from laboratory scales and compact star scales to the scale of the visible Universe. Such a unified approach may give much more definite justification of our models using all available information for the physical phenomena at all reachable scales.

The MDG model was proposed and studied in [1], [1], [1, 目]. It describes a simple generalization of the Einstein general relativity (GR), based on the following action of the gravi-dilaton sector

$$
\mathscr{A}_{g, \Phi}=\frac{c^{3}}{16 \pi G_{N}} \int d^{4} x \sqrt{|g|}(\Phi R-2 \Lambda U(\Phi))
$$

Without any relation with astrophysics and cosmology, it was studied by O'Hanlon, as early as in [D]. There the term "dilaton" for the field $\Phi$ was introduced. Formally, MDG resembles the Branse-Dicke theory with $\omega \equiv 0$, if the most important MDG-cosmological term in Eq. (메) is ignored. To some extent MDG is related with the $f(R)$ theories of gravity [ [W, 团]. In general, the $f(R)$ theories are also physically different, being only locally equivalent to MDG [ 6 ].

The values $\Phi \in(0, \infty)$ must be positive to avoid the physically unacceptable antigravity. The value $\Phi=0$ yields an infinite gravitational factor and makes the Cauchy problem in MDG not well posed [ $[0]$. The value $\Phi=\infty$ turns off the gravity and must also be excluded, as well as $\Phi=0$.

The scalar field $\Phi$ introduces a variable gravitational factor $G(\Phi)=G_{N} / \Phi=G_{N} g(\Phi)$ instead of the Newton constant $G_{N}$. The cosmological potential $U(\Phi)$ introduces a variable factor $\Lambda U(\Phi)$ instead of the constant $\Lambda$. In GR with cosmological constant $\Lambda$ we have $\Phi \equiv 1, g(\Phi) \equiv 1$, and $U(1) \equiv 1$. Due to its specific physical meaning, the field $\Phi$ has quite unusual properties.

The function $U(\Phi)$ defines the cosmological potential which must be a positive single valued function of the dilaton field $\Phi$ by astrophysical reasons. See [ [6] for all physical requirements on the cosmological potential $U(\Phi)$, necessary for a sound MDG model. There the class of withholding potentials was introduced. These confine dynamically the values of the dilaton $\Phi$ in the physical domain. It is hard to formulate such a property for the function $f(R)$ in a simple way. 


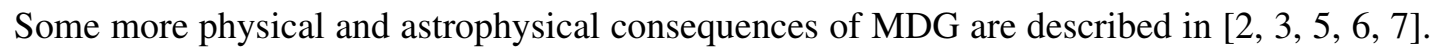
This model provides an alternative explanation of the observed astrophysical phenomena without introduction of dark energy and dark matter.

In the the present article we give a correct formulation of a star problem in MDG and consider the simplest example of a physically consistent family of SSSS for an admissible cosmological potential $U(\Phi)$. We explicitly show how the dilatonic field $\Phi$ changes the structure of the compact stars and creates a specific dilatonic sphere around them, analogous to dark matter halo.

\section{Basic equations and boundary conditions for SSSS in MDG}

In units $G_{N}=c=1$ the field equations of MDG can be written in the form:

$$
\begin{aligned}
\Phi \hat{R}_{\alpha}^{\beta}+\widehat{\nabla_{\alpha} \nabla \beta} \Phi+8 \pi \hat{T}_{\alpha}^{\beta} & =0, \\
\square \Phi+\Lambda V^{\prime}(\Phi) & =\frac{8 \pi}{3} T .
\end{aligned}
$$

Here $T_{\alpha}^{\beta}$ is the energy-momentum tensor of the matter, $\hat{X}_{\alpha}^{\beta}=X_{\alpha}^{\beta}-\frac{1}{4} X \delta_{\alpha}^{\beta}$ is the traceless part of the $4 D$-tensor $X_{\alpha}^{\beta}, X=X_{\alpha}^{\alpha}$ is its trace, the relation $V^{\prime}(\Phi)=\frac{2}{3}\left(\Phi U^{\prime}(\Phi)-2 U(\Phi)\right)$ introduces the dilatonic potential $V(\Phi)$, and the prime denotes differentiation with respect to the variable $\Phi$ [ [ $]$ ].

In the problems under consideration, the space-time-interval is $d s^{2}=e^{v(r)} d t^{2}-e^{\lambda(r)} d r^{2}-$ $r^{2} d \Omega^{2}$ [[]], where $r$ is the luminosity distance to the center of symmetry, and $d \Omega^{2}$ describes the space-interval on the unite sphere. Then, after some algebra one obtains the following basic results for a SSSS of the luminosity radius $r^{*}$.

In the inner domain $r \in\left[0, r^{*}\right]$ the SSSS structure is determined by the system:

$$
\begin{aligned}
\frac{d m}{d r} & =4 \pi r^{2} \varepsilon_{e f f} / \Phi \\
\frac{d \Phi}{d r} & =-4 \pi r^{2} p_{\Phi} / \Delta \\
\frac{d p_{\Phi}}{d r} & =-\frac{p_{\Phi}}{r \Delta}\left(3 r-7 m-\frac{2}{3} \Lambda r^{3}+4 \pi r^{3} \varepsilon_{e f f} / \Phi\right)-\frac{2}{r} \varepsilon_{\Phi}, \\
\frac{d p}{d r} & =-\frac{p+\varepsilon}{r} \frac{m+4 \pi r^{3} p_{e f f} / \Phi}{\Delta-2 \pi r^{3} p_{\Phi} / \Phi}
\end{aligned}
$$

The four unknown functions are $m(r), \Phi(r), p_{\Phi}(r)$, and $p(r)$. In Eqs. (2.2) $\Delta=r-2 m-\frac{1}{3} \Lambda r^{3}$, $\varepsilon_{e f f}=\varepsilon+\varepsilon_{\Lambda}+\varepsilon_{\Phi}, p_{e f f}=p+p_{\Lambda}+p_{\Phi}$. We obtain also two additional EOS specific for MDG:

$$
\begin{array}{llrl}
\varepsilon_{\Lambda} & =-p_{\Lambda}-\frac{\Lambda}{12 \pi} \Phi: & & \text { CEOS } \\
\varepsilon_{\Phi} & =p-\frac{1}{3} \varepsilon+\frac{\Lambda}{8 \pi} V^{\prime}(\Phi)+\frac{p_{\Phi}}{2} \frac{m+4 \pi r^{3} p_{e f f} / \Phi}{\Delta-2 \pi r^{3} p_{\Phi} / \Phi}: & & \text { DEOS } \\
\varepsilon=\varepsilon(p): & & \text { MEOS }
\end{array}
$$

Equation (2.3a) is the EOS for the cosmological energy density $\varepsilon_{\Lambda}=\frac{\Lambda}{8 \pi}(U(\Phi)-\Phi)$ and the cosmological pressure $p_{\Lambda}=-\frac{\Lambda}{8 \pi}\left(U(\Phi)-\frac{1}{3} \Phi\right)$, i.e. the cosmological EOS (CEOS). 
Equation $(2 \cdot 3 b)$ replaces the field equation $(2 \cdot d)$ and presents the dilatonic EOS (DEOS). It relates the dilatonic energy density $\varepsilon_{\Phi}=\frac{1}{8 \pi r^{2}}(\Delta / r)^{1 / 2} \frac{d}{d r}\left(r^{2}(\Delta / r)^{1 / 2} \frac{d}{d r} \Phi\right)$ and the dilatonic pressure $p_{\Phi}=-\frac{\Delta}{4 \pi r^{2}} \frac{d}{d r} \Phi$, which measures the gradient of gravitational factor.

Equation ([2.3d) presents the EOS of star matter (MEOS), see [ $[8]$ for a modern detailed survey.

Adopting the widespread assumption that the SSSS-center C ( $\Rightarrow$ index "c") is at $r_{c}=0$ we obtain the boundary conditions

$$
\begin{array}{r}
m(0)=m_{c}=0, \Phi(0)=\Phi_{c}, \quad p(0)=p_{c}, \\
p_{\Phi}(0)=p_{\Phi c}=\frac{2}{3}\left(\frac{\varepsilon\left(p_{c}\right)}{3}-p_{c}\right)-\frac{\Lambda}{12 \pi} V^{\prime}\left(\Phi_{c}\right) .
\end{array}
$$

Requiring $m_{c}=0$ we ensure finiteness of pressure $p_{c}$ simultaneously for the Newton-, GR- and MDG-SSSS. The condition on $p_{\Phi c}\left(=-\frac{2}{3} \varepsilon_{\Phi_{C}}\right)$ ensures its finiteness, being a specific MDG-centrevalue-relation: $F_{\Phi}\left(p_{\Phi c}, p_{c}, \Phi_{c}\right)=0$.

The SSSS-edge ( $\Rightarrow$ index $" * ")$ is defined by the condition $p^{*}=p\left(r^{*} ; p_{c}, \Phi_{c}\right)=0$. Then

$$
\begin{aligned}
m^{*}=m\left(r^{*} ; p_{c}, \Phi_{c}\right), \Phi^{*} & =\Phi\left(r^{*} ; p_{c}, \Phi_{c}\right), \\
p_{\Phi}^{*} & =p_{\Phi}\left(r^{*} ; p_{c}, \Phi_{c}\right) .
\end{aligned}
$$

The luminosity radius of a compact SSSS varies: $r^{*} \sim 5 \div 20 \mathrm{~km}$, depending on MEOS.

Outside the star $p \equiv 0$ and $\varepsilon \equiv 0$, and we have a dilaton-sphere, in brief $-a$ disphere. Its structure is determined by the shortened system (․2. i.e., the Eq. (․2d) has to be omitted. For the exterior domain $r \in\left[r^{*}, r_{U}\right]$ we use Eqs. (ㅁ.5) as left boundary conditions. The right boundary is defined by the cosmological horizon $r_{U}: \Delta\left(r_{U} ; p_{c}, \Phi_{c}\right)=0$, where the de Sitter vacuum is reached: $\Phi\left(r_{U} ; p_{c}, \Phi_{c}\right)=1$. Thus, we obtain a new MDG-centre-value-relation: $F_{\Lambda}\left(p_{c}, \Phi_{c}\right)=0$.

Hence, in MDG, as well as in the Newton gravity and GR, we have a one-parameter-family of SSSS, because of the existence of the two MEOS-dependent-relations:

$$
F_{\Phi}\left(p_{\Phi c}, p_{c}, \Phi_{c}\right)=0, F_{\Lambda}\left(p_{c}, \Phi_{c}\right)=0 .
$$

The observable value $\Lambda \approx 1.27 \times 10^{-44} \mathrm{~km}^{-2}$ is very small. As a result, the luminosity radius of the Universe $r_{U} \sim 1 / \sqrt{\Lambda} \sim 10^{22} \mathrm{~km}$ is very large in comparison with $r^{*} \sim 10 \mathrm{~km}$.

Further on, we use the cosmological potential $U(\Phi)=\Phi^{2}+\frac{3}{16 \mathfrak{p}^{2}}(\Phi-1 / \Phi)^{2}$. Hence, $V^{\prime}(\Phi)=$ $\frac{1}{2 \mathfrak{p}^{2}}\left(1-1 / \Phi^{2}\right)$. For useful comments and a more general form of the admissible cosmological potentials $U(\Phi)$ see [ [G, 目]. The parameter $\mathfrak{p}=\sqrt{\Lambda} \hbar / c m_{\Phi}$ is the dimensionless Compton length (measured in cosmological units) of the dilaton $\Phi$. MDG is consistent with observation if $\mathfrak{p} \lesssim 10^{-30}$ $\left(m_{\Phi}>10^{-3} \mathrm{eV} / c^{2}\right)$ [ [], []]. If one takes as a dilaton the only currently known fundamental scalar particle, the Higgs boson with mass $m_{H} \approx 125 \mathrm{GeV} / \mathrm{c}^{2}$, then $\mathfrak{p} \approx 1.8 \times 10^{-43}$. Further on, we use a non-realistic big value $\mathfrak{p}=10^{-21}$ (the Compton length $\sim 9 \mathrm{~km}$ ) for a more transparent graphical representation of the results.

\section{Results for the simplest MEOS of neutron matter}

The most idealized MEOS of neutron matter is the one from the GR-TOV model [ [ $]$ ]: $\varepsilon=$ $\frac{1}{4 \pi} x(\sinh t-t), p=\frac{1}{12 \pi}(\sinh t-8 \sinh (t / 2)+3 t)$. It describes the ideal Fermi neutron gas at zero temperature and facilitates our study of the pure-MDG-effects in SSSS, shown in Figs. ㅁ․ 


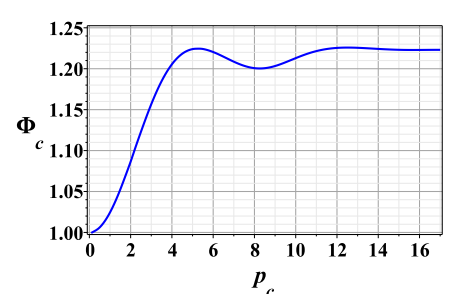

Figure 1: The specific MDG-curve $F_{\Lambda}\left(p_{c}, \Phi_{c}\right)=0$

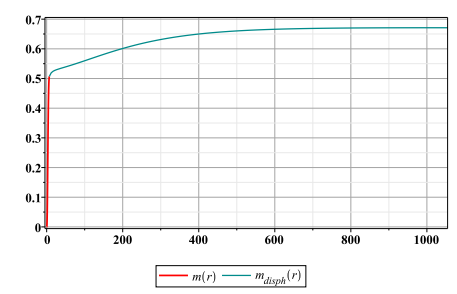

Figure 3: The disphere-mass dependence on $r$

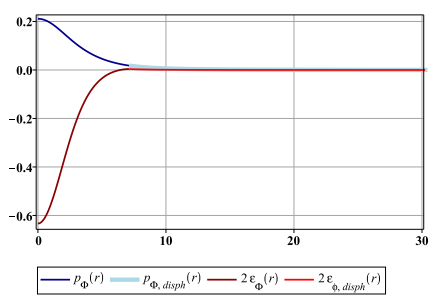

Figure 5: The dependencies $2 \varepsilon_{\Phi}(r)$ and $p_{\Phi}(r)$ in accord with DEOS

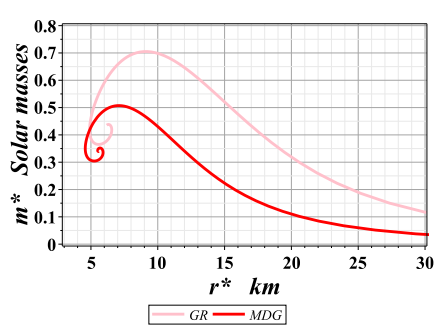

Figure 7: Mass - radius relations: in GR $\left(m_{\text {max }}^{*} \approx .7051 m_{\odot}, r_{\text {max }}^{*} \approx 9.209 \mathrm{~km}\right)$ and in $\operatorname{MDG}\left(m_{\text {max }}^{*} \approx .5073 m_{\odot}, r_{\text {max }}^{*} \approx 7.092 \mathrm{~km}\right)$
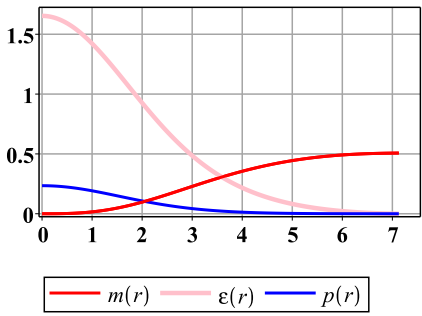

Figure 2: The MDG-SSSS interior in accord with MEOS

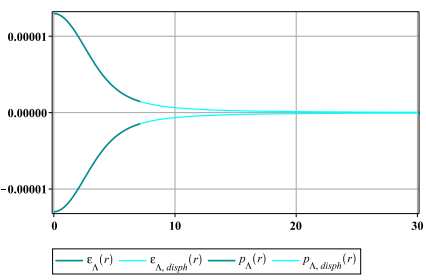

Figure 4: The dependencies $\varepsilon_{\Lambda}(r)$ and $p_{\Lambda}(r)$ in accord with CEOS

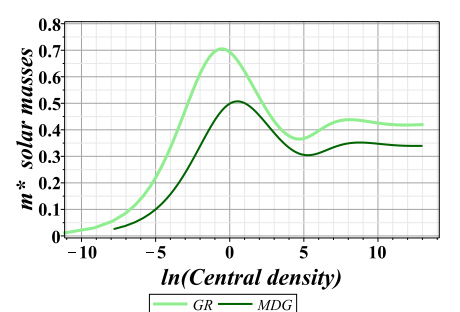

Figure 6: The $\mathrm{m}^{*}-\boldsymbol{\varepsilon}_{c}$ dependencies

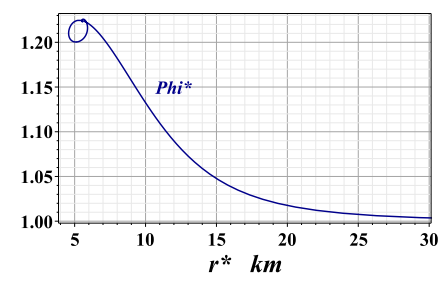

Figure 8: The specific MDGdependence $\Phi^{*}(r *)$

As seen in Fig. Z, MDG-SSSS are lighter and more compact than GR-SSSS. As in GR, MDG-

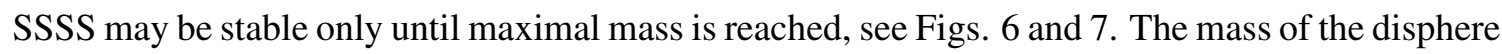
$m_{\text {disp }}(r)$ outside MDG-SSSS exponentially goes to a constant: $m_{\text {disp }} \approx .1638 M_{\odot}$. The total mass of the object $m_{\text {total }} \approx 0.671 M_{\odot}$ is quite close to the mass of GR-SSSS $m_{G R, \max } \approx 0.705 M_{\odot}$.

An interesting problem is a model of a moving and rotating star in MDG. In it one can expect an asymmetric configuration with appearance of different centers of the star and its disphere, or 


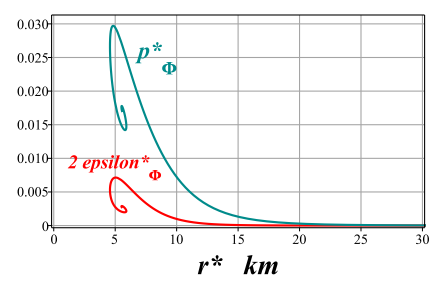

Figure 9:

The MDG-dependences $2 \varepsilon_{\Phi}^{*}(r *), p_{\Phi}^{*}(r *)$. Maximal values are reached for smaller $r *$ than in Fig. $\square$

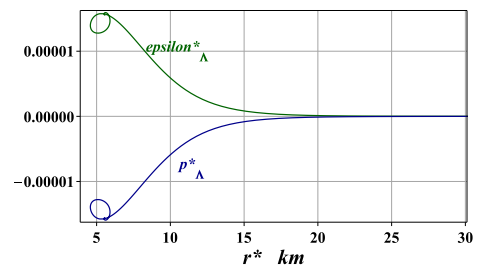

Figure 10:

The specific MDG-dependences $\varepsilon_{\Lambda}^{*}(r *)$ and $p_{\Lambda}^{*}(r *)$ in accord with CEOS

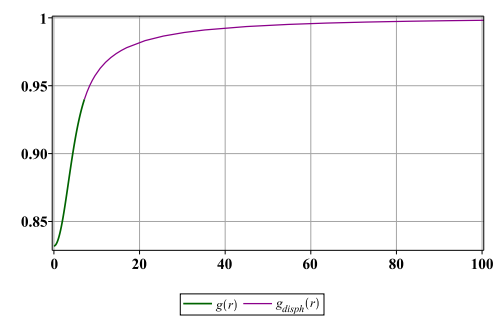

Figure 11: Unexpectedly large variations of the gravitational factor $g(r)=1 / \Phi(r)$ in MDG-SSSS

even detachment of the parts of disphere. For similar effects at cosmological scales see [Q].

This research was supported in part by the Foundation for Theoretical and Computational Physics and Astrophysics and Grant of the Bulgarian Nuclear Regulatory, Agency for 2014, as well as by "NewCompStar", COST Action MP1304.

\section{References}

[1] A. A. Starobinsky, Phys. Lett. B 9199 (1980); V. Faraoni, Phis Rev. D 74104017 (2006); A. A. Starobinsky, JETP Lett. 86157 (2007); T. P. Sotiriou, V. Faraoni, Rev. Mod. Phys. 82451 (2010); A. De Felice, S. Tsujikawa, Living Rev. Rel. 133 (2010); S. Nojiri, S. D. Odintsov, Phys. Rept. 505 59-144 (2011); T. Clifton, P. G. Ferreira, A. Padill, C. Skordis, Physics Reports 5131 (2012).

[2] O’Hanlon, Phys. Rev. Lett. 29137 (1972).

[3] P. P. Fiziev, Mod. Phys. Lett. A, 151077 (2000).

[4] A. V. Frolov, PRL 101061103 (2008); T. Kobayashi and K. Maeda, Phys. Rev. D 78064019 (2008); E. Babichev, D. Langlois, Phys. Rev. D 80, 121501 (2009); E. Babichev, D. Langlois, Phys. Rev. D 81, 121051 (2010). A. S. Arapoglu, C. Deliduman, K. Y. Eksi, J. Cosmology and Astroparticle Physics, 07020 (2011); C. Deliduman, K. Y. Eksi, V. Keles, J. Cosmology and Astroparticle Physics, 05036 (2012); A. V., Astashenok, S. Capozziello, S. D. Odintsov, arXiv:1401.4546 [gr-qc].

[5] Fiziev P. P., Yazadjiev S., Boyadjiev T., Todorov M., Phys. Rev. D 61124018 (2000); Plamen P. Fiziev, arXiv:gr-qc/0202074; P. Fiziev, Georgieva D., Phys. Rev. D 67064016 (2003).

[6] P. P. Fiziev, Phys. Rev. D 870044053 (2013).

[7] G. Esposito-Farese, D. Polarski, Phys. Rev. D 63063504 (2001).

[8] L. Rezzola, O. Zanotti, em Relativistic Hydrodynamics, Oxford University Press (2013).

[9] D. Clowe, M. Bradac,A. H. Gonzalez, M. Markevitch, S. W. Randall, C. Jones, D. Zaritsky, The Astrophysical Journal, 648 L109 (2006). 